\title{
Application of Graph Theory Features for the Objective Diagnosis of Depressive Patients with or without Anxiety: an Rs-fMRI Study
}

\author{
Xiang-Yu SHEN ${ }^{1}$, Jing-Yu ZHU ${ }^{1}$, Mao-Bin WEI ${ }^{1}$, Jiao-Long QIN $^{1}$, \\ Rui YAN ${ }^{2}$, Qiu-Xiang WEI ${ }^{2}$, Zhi-Jian YAO ${ }^{2,3}$, Qing LU', * \\ ${ }^{1}$ Key Laboratory of Child Development and Learning Science (Ministry of Education), \\ Research Centre for Learning Science, Southeast University, Si Pailou 2, \\ Nanjing,210096, China \\ ${ }^{2}$ Department of Psychiatry, Affiliated Nanjing Brain Hospital of Nanjing Medical \\ University, No. 264 Guangzhou Road, Nanjing, 210029, China \\ ${ }^{3}$ Nanjing Brain Hospital, Nanjing University Medical School, 22 Hankou Road, Nanjing, \\ 210093, China \\ ${ }^{*}$ Corresponding author
}

Keywords: Rs-fMRI, MDD, Anxiety, Graph theory, Machine learning.

\begin{abstract}
Purposes: To probe abnormality that may lead to anxiety in depressive patients. Procedures: This study investigated the graph theory features ahead of machine learning feature selection procedure. Classification methods were applied afterwards. Methods: Graph theory, statistical analysis and forward sequential feature selection were combined to find features. SVM classifier was also involved. Results: 1 global and 22 local features were found correlated with clinical anxiety factor. Conclusions: Anxiety is correlated with emotion and cognitive loop and other regions.
\end{abstract}

\section{Introduction}

Anxious depression is a common clinical subtype of major depressive disorder(MDD) [1]. Dysphoric mood, disturbed sleep, somatic complaints, altered interoceptive awareness, and increased morbidity are all anxiety characters [2-4]. Many resting state fMRI (rs-fMRI) studies have been searching for biomarker that can distinguish anxious and non-anxious depressive patients[5]. However, despite the plenty works features of graph theory were not completely discussed.

In present study, we involved in 22 non-anxious depressive patients and 21 depressive patients, abstracting 7 global and 9 local graph theory features of each individual as a primary feature vector. And then the combined feature selection method was applied to efficiently reduce the dimension of the features. Statistical analysis and classification was performed afterwards.

\section{Methods}

\section{Subjects}

Since this study focused on the anxiety in depressive patients, healthy control was not included [6]. Forty-three patients with MDD were recruited (including 22 patients without anxiety and 21 patients with anxiety). The initial diagnoses of depression were made according to DSM-IV. All the patients were included according to the criteria: (1) the Hamilton depression scale (HAMD) scores were over 17, (2) medicine naive for at 
least 2 weeks, (3) without history of drug abuse or alcohol dependency, (4) no other mental illness, (5) anxiety/somatization factor scores below 7 were assigned as without anxiety, while anxiety/somatization factor scores over 7 were assigned as with anxiety. The 17-item HAMD Anxiety /Somatization factor includes six items: Anxiety (psychic), Anxiety (somatic), Somatic Symptoms (gastrointestinal), Somatic Symptoms (general), Hypochondriasis and Insight.

The study was approved by the Research Ethics Review Board of Nanjing Brain hospital in China. The demographic characteristics of the subjects were summarized in Table1.

Table 1. Demographic and Clinical data of the groups

\begin{tabular}{|l|l|l|l|}
\hline \multicolumn{1}{|c|}{ Item/Group } & \multicolumn{1}{c|}{ Non-anxious } & \multicolumn{1}{c|}{ Anxious } & \multicolumn{1}{c|}{$p$} \\
\hline Age & $33.7 \pm 8.05$ & $32.6 \pm 8.95$ & 0.671 \\
\hline Education & $13.5 \pm 2.92$ & $13.7 \pm 2.50$ & 0.800 \\
\hline Anxiety/somatization factor & $5.91 \pm 1.54$ & $9.14 \pm 1.39$ & $0.0001 *$ \\
\hline HAMD & $24.5 \pm 2.52$ & $25.9 \pm 3.30$ & 0.136 \\
\hline Subject number & 22 & 21 & \\
\hline
\end{tabular}

\section{Data Acquisition and Preprocessing}

All imaging data were collected by a Siemens verio scanner. Before the scans, subjects were instructed to close their eyes and not to think systematically. Image preprocessing was completed by the Data Processing Assistant for Resting-State fMRI (DPARSF) toolbox [7] and the SPM8 package (http://www.fil.ion.ucl.ac.uk/spm).

Functional connectivity matrixes were extracted by the DPARSF analyzing steps using AAL template. The matrixes were sparse where the sparsity was determined by scaling from 0.1 to 0.9 with a step as 0.05 , and making a permutation test of the two groups divided by clinical anxiety factor 7 . Since the optimal sparsity of each individual differed from each other and small changes ranging from 0.3 to 0.6 of it may not lead to sudden deteriorate of the primary graph feature performance, the sparsity was determined by all members together and was not involved in the LOO strategy. The sparsity led to the least p-value (which represent the highest statistical significant between the two groups) should be optimal.

\section{Computation of Graph Theory Features}

We computed 7 global and 9 local graph theory features of each individual. Global measures representing the integration situation have only one value for each graph whereas local measures representing segregation signature have $n$ values ( $n$ is the number of nodes in the graph) for each graph [8]. Six functional integration graph features were estimated, which are characteristic path length [9], global efficiency [10], density, density edge number, network walk and transitivity scalar [11]. Another global feature assortativity was an assessment of resilience. Three segregation graph features were also calculated, which are clustering coefficient [9], nodal efficiency and local efficient [10]. Six other nodal measures including nodal degree, nodal strength, eccentricity, nodal betweenness centrality vector, eigenvector centrality and walk length distribution [12], were applied to assess properties of brain regions. We used the Brain Connectivity Toolbox (www.brain-connectivity-toolbox. net). And the final feature number of each individual was 1051 ( 7 global $+116 \times 11$ local $)$. 


\section{Statistical Analysis and Feature Selection}

We took a permutation test of each feature and kept the features which showed significant discriminative power (with the resulted p-value of permutation test below 0.05). And then a Fisher coefficient feature selection was taken, which denotes the discriminative power of one feature [8]. The performance was estimated by the accuracy the classifier gained by adding features.

\section{Classification and Correlation Test}

Classification method was applied to the remaining subset of features in feature selection procedure. We chose supervised classifier SVM using a Leave-One-Out (LOO) method to confirm the generalization of this classification process.

Correlation test was also applied to probe the ability these features possess to differentiate depressive patients with or without anxiety.

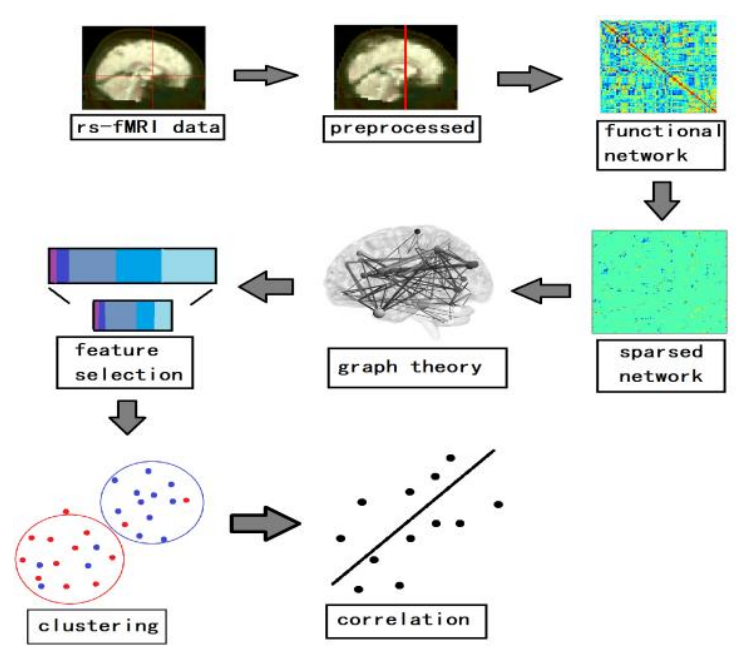

Figure 1. All the procedurals in this work

\section{Results}

\section{Brain Network and Sparsity Decision}

The data preprocessing, feature selection and classification process is shown in Fig. 1. Functional connectivity was calculated as correlation between AAL brain regions as the edges of brain network. And the resulted p-value changing with the sparsity was shown in Fig. 2. As is shown in the figure, the least p-value was resulted using sparsity 0.5. Thus we chose 0.5 as the optimal sparsity.

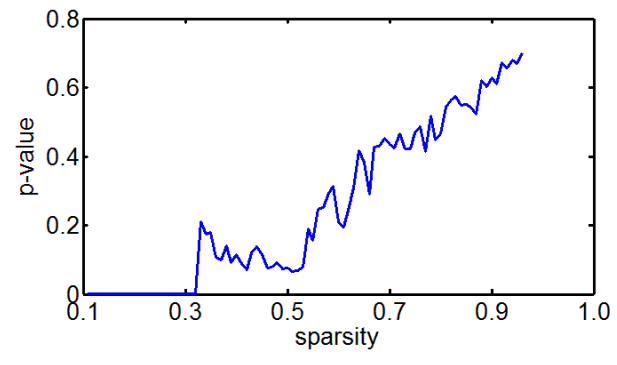

Figure 2. The p-value changes with sparsity.

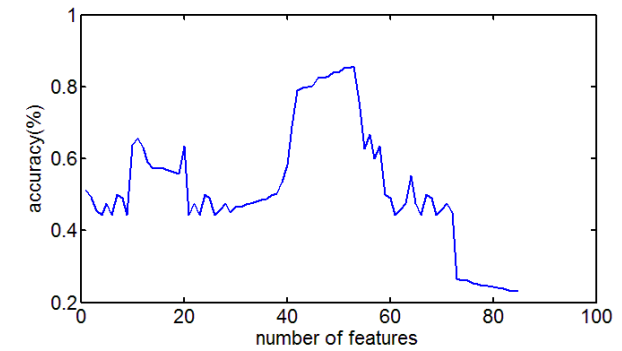

Figure 3. The accuracy changes in one LOO loop. 
Table 2. The remaining features after the two feature selection procedure.

\begin{tabular}{|l|l|c|}
\hline \multicolumn{1}{|c|}{ local graph feature } & \multicolumn{1}{c|}{ brain region } & discriminative power \\
\hline eigenvector centrality & Frontal_Sup_Medial_L & 0.512438 \\
\hline nodal strength & Frontal_Sup_Medial_L & 0.491747 \\
\hline clustering coefficient & SupraMarginal_R & 0.460903 \\
\hline nodal betweenness centrality vector & SupraMarginal_R & 0.460903 \\
\hline clustering coefficient & Rolandic_Oper_L & 0.442652 \\
\hline nodal betweenness centrality vector & Rolandic_Oper_L & 0.442652 \\
\hline eigenvector centrality & Temporal_Pole_Mid_R & 0.429753 \\
\hline eigenvector centrality & Cingulum_Ant_R & 0.427005 \\
\hline eigenvector centrality & Frontal_Mid_Orb_L & 0.423196 \\
\hline eigenvector centrality & Fusiform_R & 0.416208 \\
\hline eigenvector centrality & Frontal_Mid_Orb_R & 0.413367 \\
\hline clustering coefficient & Pallidum_L & 0.409213 \\
\hline nodal betweenness centrality vector & Pallidum_L & 0.409213 \\
\hline clustering coefficient & Thalamus_L & 0.393346 \\
\hline nodal betweenness centrality vector & Thalamus_L_ & 0.393346 \\
\hline eccentricity & Cingulum_Post_L & 0.391986 \\
\hline
\end{tabular}

\section{Feature Selection}

The feature selection results were shown in Table 2. Only top $10 \%$ features were listed. The discriminative power was the mean value of the LOO repeat times. Only one global feature was found discriminative, which is global efficiency in Superior Frontal gyrus with the discriminative power as 0.07875 . And 155 local nodal features of were found significant to the anxiety in depressive patients. Afterwards the classification accuracy achieved by adding features was shown in Fig. 3 .

\section{Classification Performance}

By applying the LOO procedure the classification performance was able to be quantified. The percentage of false assignment of labels was counted to evaluate the accuracy of this classification procedure. The predictive accuracy, the sensitivity and the specificity was $0.8372,0.8571$, and 0.8182 respectively.

\section{Correlation between Clinical Factor and Graph Features}

The correlation of clinical factor and graph features were shown below in Table 3 . The discriminative brain regions lie more in emotion-cognitive loop.

\section{Discussion}

In this study, we constructed a feature matrix using 7 global and 9 local graph theory features. We found out distinguishing features with a classification predictive accuracy as 0.8372 and the sensitivity as 0.8571 , the specificity as 0.8182 .

Intriguingly, the graph features correlated with anxiety factor we found lay mostly in emotion-cognitive loop. The Hippocampus regions and amygdala regions are believes to be necessary for the establishment of cognition and emotion, whose dysfunction may relate to the anxiety symptom [13]. It has been proved that the right prefrontal activation is associated with certain forms of anxiety [14]. Waugh et al. found out activation in temporal gyrus (during task recovery), and less activation of the cerebellum among 
anxious patients [15]. The Andreescu et al's work suggest that anxiety maintain a "higher alert," scanning both in occipital areas and parietal areas [6]. Therefore, the abnormal observed in our study was believed to indicate impairments in intro-spective integration processing in anxious depression.

The feature selection procedure including statistical analysis and machine learning method was time consuming and efficient, since the features performed well in the classification and consistency measurements as shown in Fig. 3. Moreover, the discriminative power in our study can be quantified after the feature selection (Table 3).

Table 3. Correlation of clinical factor and graph features

\begin{tabular}{|c|c|c|c|}
\hline $\begin{array}{l}\text { Local graph } \\
\text { features } \\
\end{array}$ & Brain regions & $\begin{array}{l}\text { Correlation } \\
\text { coefficient }\end{array}$ & $\begin{array}{c}\text { Statistical } \\
\text { significance }\end{array}$ \\
\hline \multirow{3}{*}{ degree } & Cingulum_Ant_L & -0.314 & 0.016 \\
\hline & Occipital_Sup_L & 0.307 & 0.019 \\
\hline & Occipital_Sup_R & 0.405 & 0.002 \\
\hline \multirow{2}{*}{$\begin{array}{l}\text { clustering } \\
\text { coefficient }\end{array}$} & Supp_Motor_Area_R & -0.290 & 0.027 \\
\hline & Frontal_Sup_Medial_L & -0.325 & 0.013 \\
\hline \multirow{2}{*}{ nodal efficiency } & Occipital_Sup_L & 0.263 & 0.046 \\
\hline & Occipital_Sup_R & 0.372 & 0.004 \\
\hline \multirow{10}{*}{ eccentricity } & Cingulum_Post_L & 0.333 & 0.011 \\
\hline & Hippocampus_R & 0.356 & 0.006 \\
\hline & Amygdala_L & 0.272 & 0.039 \\
\hline & Calcarine_L & 0.338 & 0.009 \\
\hline & Lingual_R & 0.317 & 0.015 \\
\hline & Occipital_Sup_L & 0.352 & 0.007 \\
\hline & Occipital_Inf_R & 0.317 & 0.015 \\
\hline & Temporal_Mid_L & 0.274 & 0.037 \\
\hline & cerebellum & 0.327 & 0.012 \\
\hline & cerebellum & 0.281 & 0.033 \\
\hline \multirow{2}{*}{$\begin{array}{l}\text { nodal betweenness } \\
\text { centrality vector }\end{array}$} & Supp_Motor_Area_R & -0.290 & 0.027 \\
\hline & Frontal_Sup_Medial_L & -0.325 & 0.013 \\
\hline $\begin{array}{l}\text { eigenvector } \\
\text { centrality }\end{array}$ & Occipital_Sup_L & 0.280 & 0.033 \\
\hline \multirow{2}{*}{ nodal strength } & Occipital_Sup_L & 0.347 & 0.008 \\
\hline & Occipital_Sup_R & 0.378 & 0.003 \\
\hline
\end{tabular}

\section{Conclusions}

In present work we applied an advanced feature selection method on graph features, and result in fine accuracy of differing anxious depressive patients with nonanxious depressive patients. The discriminative features mostly lay in emotion-cognitive loop, which we suggest played an important role in the depressive anxiety machanism.

\section{Acknowledgement}

The work was supported by the grants of: National High Technology Research and Development Program of China (863 Program) (2015AA020509); The National Natural Science Foundation of China (81571639,81371522, 61372032); Jiangsu Clinical Medicine Technology Foundation (BL2014009); Jiangsu Natural Science Foundation (BK20131074). 


\section{References}

[1] Rush AJ. The varied clinical presentations of major depressive disorder. J Clin Psychiatry. 2007;68 Suppl 8:4-10.

[2] Fava M, Rush AJ, Alpert JE, Balasubramani GK, Wisniewski SR, Carmin CN, et al. Difference in treatment outcome in outpatients with anxious versus nonanxious depression: a STAR*D report. Am J Psychiatry. 2008;165:342-51.

[3] Ionescu DF, Niciu MJ, Mathews DC, Richards EM, Zarate CA, Jr. Neurobiology of anxious depression: a review. Depress Anxiety. 2013;30:374-85.

[4] Lydiard RB, Brawman-Mintzer O. Anxious depression. J Clin Psychiatry. 1998;59 Suppl 18:10-7.

[5] Liu CH, Ma X, Song LP, Fan J, Wang WD, Lv XY, et al. Abnormal spontaneous neural activity in the anterior insular and anterior cingulate cortices in anxious depression. Behavioural Brain Research. 2015;281:339-47.

[6] Andreescu C, Wu M, Butters MA, Figurski J, Reynolds CF, 3rd, Aizenstein HJ. The default mode network in late-life anxious depression. Am J Geriatr Psychiatry. 2011;19:980-3.

[7] Chao-Gan Y, Yu-Feng Z. DPARSF: A MATLAB Toolbox for "Pipeline" Data Analysis of Resting-State fMRI. Front Syst Neurosci. 2010;4:13.

[8] Khazaee A, Ebrahimzadeh A, Babajani-Feremi A. Application of advanced machine learning methods on resting-state fMRI network for identification of mild cognitive impairment and Alzheimer's disease. Brain Imaging Behav. 2015.

[9] Watts DJ, Strogatz SH. Collective dynamics of 'small-world' networks. Nature. 1998;393:440-2.

[10] Latora V, Marchiori M. Efficient behavior of small-world networks. Phys Rev Lett. 2001;87:198701.

[11] Goni J, van den Heuvel MP, Avena-Koenigsberger A, de Mendizabal NV, Betzel RF, Griffa A, et al. Resting-brain functional connectivity predicted by analytic measures of network communication. Proceedings Of the National Academy Of Sciences Of the United States Of America. 2014;111:833-8.

[12] Sporns O. Small-world connectivity, motif composition, and complexity of fractal neuronal connections. Biosystems. 2006; 85:55-64.

[13] Davidson RJ. Anxiety and affective style: role of prefrontal cortex and amygdala. Biol Psychiatry. 2002; 51:68-80.

[14] Davidson RJ, Abercrombie H, Nitschke JB, Putnam K. Regional brain function, emotion and disorders of emotion. Curr Opin Neurobiol. 1999;9:228-34.

[15] Waugh CE, Hamilton JP, Chen MC, Joormann J, Gotlib IH. Neural temporal dynamics of stress in comorbid major depressive disorder and social anxiety disorder. Biol Mood Anxiety Disord. 2012; 2:11. 